\title{
Communication technologies in the study environment: institutional and personal media as a reflection of organisational structure
}

\section{Anne Mette Thorhauge}

MedieKultur 2012, 53, 22-36

Published by SMID | Society of Media researchers In Denmark | www.smid.dk The online version of this text can be found open access at www.mediekultur.dk

In this article, I will analyse and discuss two qualitative case studies concerning ICT in the study environment at the University of Copenhagen, Denmark. I will place special focus on the way in which organisational perspectives as well as technological affordances shape how communication technologies are integrated into organisational structures and practices on campus. This involves a comparison between course management systems on the one hand and students' personal media (mobile phones, e-mails) on the other hand, with regard to how these are used on campus. On the basis of this analysis, I will argue that the ways in which these technologies are used reflects two different perspectives on the interplay between communication technology and organisational structure: organisational structure as an anticipation of communication patterns implied in course management system's design and implementation as well as organisational structure as a product of the use of personal media.

\section{Introduction}

Like so many other university lecturers, my organisation has provided me with a communication tool, a CMS or "course management system", that allows me to communicate more efficiently with my students. With this, I can make announcements and distribute texts fairly easily, and I am rather happy with the system. However, a quick look at the usage 
statistics tells me that the students are not quite as enthusiastic. Whereas some students frequently log into the system, others seem to avoid it as much as possible and get their information elsewhere. For this reason, I have become accustomed to asking key students to distribute particularly important messages in their informal network (their Facebook group) to ensure that everyone gets to know in time. I am also aware that the students have a common Dropbox folder where they redistribute texts I upload on the CMS. Apparently, this is a more flexible and natural solution for them than looking it up on the CMS. And this is not necessarily a problem as long as my texts and announcements reach those they need to reach. The real problem emerges when I ask the students to share their own drafts, links, or opinions by way of the CMS in order to make it available to the entire class - and when the students simply refuse. For some reason, they are very reluctant to do this. They seem to consider the CMS to be "the teacher's medium" and generally ignore the many-to-many communication facilities that are also included in the system.

This partly has to do with the way students deal with each other and the particular sort of work environment that the university campus represents. As I argue below, students do not necessarily consider their fellow students to be colleagues, and they may thus relate more to the teacher as a relevant source of information and ignore each other. However, as their use of Facebook and Dropbox indicates, they do to some degree share files and communicate among each other concerning relevant study issues, yet they tend to avoid using the CMS for this purpose. It does not seem to suit their communication practices. This reflects a more general issue regarding communication technologies in organisations and work environments: It is very difficult to map the communication structures and informal networks composing the everyday reality of organisational life, and the intranet, content management systems, and similar technologies often remain rather segregated from the everyday organisational reality they are meant to reflect.

In the following sections, I will discuss this issue on the basis of two qualitative case studies focusing on ICT in the study environment at the University of Copenhagen's Southern Campus. I will analyse the way in which the students in my study communicate and use media in their everyday activities, and I will show how the institutional integration and technological affordances of particular communication technologies cause them to reflect different aspects of organisational life. On the basis of this analysis, I will argue that the interplay between communication technologies and organisational structure can be approached from two different analytical perspectives. On the one hand, the design and implementation of communication technologies can be seen as an attempt to map the communication practices of a given organisation. On the other hand, the actual use of communication technologies reflects the communicative relationships as they exist among individuals within the organisation. Whereas the former reflects the organisational structure at a given point in time, the latter reflects organisational structure as a process. Due to certain technological characteristics, the personal media in this case study more directly reflects the organisational structure as a product of continuous interaction, and I will argue 
that a greater focus on these characteristics may help create designs that bridge the gap between communication systems and everyday practices.

\section{Communication in organisations: organisations as communication}

Deetz (1995) distinguishes between three different ways of defining organisational communication: as something conducted by a communication department, as something taking place within organisations, and as a means of explaining organisations. From the latter perspective, organisations are regarded as communicative phenomena constructed and reconstructed by the communication of their members. This view of organisations has received considerable attention in recent years (Cooren, Taylor, \& Van Every, 2005; Fairhurst \& Putnam, 2004; Taylor \& Robichaud, 2004; Taylor \& Van Every, 2000), and it represents a basic assumption in the present case studies. However, organisations as communicative phenomena can be explained in many ways, directing focus toward different aspects of the organisation.

McPhee $(1985,2004)$ proposes a structurational view of communication and organisational structures, defining formal structure as "the various systems for decision, support, management information, work evaluation and compensation, and financial control" (McPhee, 1985, p. 149) and further states that "the very existence of structure is dependent on its communication in a formal, explicit, authoritative way, often in writing in a document that has recognised status [...] in the organisation" (ibid., p. 160). This type of communication is termed "structure communication" and can be described as indirect communication replacing direct dialogue and as an authoritative meta-communication describing the communication and interaction processes of the organisation. In this way, McPhee explains the relationship between formal structure and communication by defining a specific genre of organisational communication and by emphasising the character of texts as symbolic representations. In terms of the case studies presented in this article, one example could be the course plan defining the times, dates, and themes of course communication during the term. Another example could be the CMS course room assigning different users with different roles (students, teachers, administrators) and communicative privileges in accordance with the general organisational structure.

In contrast, Taylor et al. (Taylor, Cooren, Giroux, \& Robichaud, 1996; Taylor \& Robichaud, 2004) focus on the relationship between organisational structure and communication in general. More specifically, they see the relationship between organisational structure and communication as a process in which text is continually translated into conversation, and conversation is continually translated into text. By text, Taylor et al. mean the material realisation of communication by way of the human voice or other media. By conversation, they mean the action implied by this communication (pp. 7-8). Taylor et al. base this distinction on Austin's speech act theory. In Austin's terms, text refers to the locutionary aspect of a statement - the statement as an utterance - whereas conversation refers to the illucutionary aspect of a statement - the statement as social action (p. 11). The latter includes 
the situational characteristics - "certain persons in certain circumstances" - that grant the statement its "illocutionary force". Taylor et al. thus suggest that "the missing components in a locutionary [...] explanation of communication which are successfully incorporated in an illocutionary/perlocutionary view, are precisely what we usually mean by organisation" (p. 12). Organisational structures are therefore those basic assumptions about roles and situations that give a locutionary statement its illocutionary force and that are continually reconfirmed or negotiated through the process of communication. In later writings, Taylor et al. add the concept of co-orientation as an important aspect of this process (Taylor, 2006; Taylor \& Robichaud, 2004). Co-orientation concerns the continuous establishment of compatible beliefs and coordinated responses (Taylor, 2006), thereby representing the "practical outcome of conversation" (Taylor \& Robichaud, 2004). In other words, orientation toward a common reality is the product of the organising activities. In terms of the case studies presented in this article, an example could be the way in which students and teachers continuously confirm and uphold particular roles and relationships by communicating in accordance with the conventions they prescribe, establishing a common understanding of the activity of studying and what it involves.

In this light, the interplay between communication and organisational structure can be seen as embedded in either particular communication genres or in the general practice of everyday communication. In both cases, the involvement of particular communication technologies may affect the process and the way in which roles and routines are implemented and, accordingly, how they develop within the context of the organisations. This article aims to describe how the integration of different types of communication technologies may affect this process.

\section{Media and technology in (educational) organisations}

As is the case with the role of communication in organisations, the concept of technology in organisations can be approached from several perspectives. Wanda Orlikowski (1992) distinguishes between three research traditions concerning this. A group of early works (Aldrich, 1972; Blau, Falbe, McKinley, \& Tracy, 1976) assume technology to be an objective force with deterministic effects on organisations whereas a later group of works (Bijker, Hughes, \& Pinch, 1987) focuses on technology as a product of shared interpretations and interventions. While the first group fails to account for the significance of human choice and action, the second groups fails to account for the material properties of technology. However, a more recent group of works sees technology as a "trigger of structural change [...] an intervention into the relationship between human agents and organization structure, which potentially changes it" (Orlikowski, 1992, p. 402). Working from this perspective, Orlikowski establishes a conceptual framework for understanding technology in organisations on the basis of Giddens' theory of structuration. Orlikowski defines two basic characteristics of a technology: its duality and its interpretive flexibility. Regarding duality, technology is physi- 
cally constructed by human beings in a social context at the same time as it mediates human action and communication by way of its material properties. Furthermore, technology tends to become institutionalised as part of the structural properties of the organisation. This duality has consequences for the technology's interpretive flexibility. Being a manmade artefact, technology is open to interpretations while, at the same time, the material characteristics of a given technology as well as its institutional context place certain limitations on interpretation. Orlikowski furthermore points out that the technology's material and structural properties are realised in the context of its use (Orlikowski, 2000). A given technology may have a wide range of structural properties embedded in its design, but it is only when a user chooses to enact certain aspects of this technology at the expense of other aspects that it turns into an institutional reality that limits and enables action in particular ways.

In the context of technology in a study environment, this means that although course management systems and other communication technologies may involve a variety of possible functionalities, it is the specific user groups in specific institutional contexts that define what is realised with and what remains a latency of the technology. Course management systems, for instance, often involve rather simple tools for mass distribution of course materials as well as more advanced tools for interactive learning, but it is the students and educators within particular institutional contexts who enact and appropriate particular aspects of this technology. Thus, even though CMS is a rather widespread technology in educational institutions today (McGill \& Hobbs, 2008; Paulsen, 2003; Selwyn, 2007), its use remains limited (Lonn \& Teasley, 2009; Sclater, 2008; Selwyn, 2007). In a study on perceptions and uses of learning management systems, Lonn and Teasly (2009) conclude that both students and teachers tend to prefer time-saving functionalities to functionalities that improve teaching and learning. This is in line with a more general critique of these systems' inflexibility and their tendency to embody institutional practices and exclude potentials for innovation offered by new web 2.0 technologies (Sclater, 2008). In accordance with Orlikowski's analytical framework, this may well be due to the particular institutional context, including academic identities (Hanson, 2009) and teaching practices (Lonn \& Teasley, 2009; Selwyn, 2007). The technology is taken into use in accordance with existing communication and teaching practices that define what are considered to be relevant and less relevant functionalities. It is unlikely that the technology will change anything as long as these practices remain. That would presuppose a more focused pedagogical effort (for instance, see Grønning, 2011).

The case studies presented in this article add another perspective to the discussion by comparing several communication technologies available on campus. Whereas studies of technology in organisations often focus on single technologies, communication on campus is potentially supported by a multitude of communication technologies suiting different communication purposes. Personal media and social media offer alternatives to the course management system, and students may choose these for various reasons. In the following sections, I will argue that personal media such as cell phones and e-mail offer a more obvious choice to students due to their less institutionalised and less professionalised charac- 
ter (Lüders, Proitz, \& Rasmussen, 2010; Lüders, 2008). Similarly, compared with the course management system, social media represent a fundamentally different way of visualising social networks and making them available (Boyd \& Ellison, 2008). I will argue that these characteristics - which are due partly to their particular institutional integration and partly to particular material and designed aspects of the technologies - make them reflect very different aspects of organisational life.

\section{Methodological approach}

In accordance with this theoretical framework, the two studies have focused on how students communicate on campus, how these communication practises reflect the organisational structure of the study environment, and how the course management system as well as other communication technologies are involved in this process. However, this interplay between communication practices, organisational structure, and communication technologies has been approached from different perspectives. The first case study was initiated in the summer of 2007 and initially focused on on-campus activities and the communication facilities offered by the university administration. That is, it asked how communication took place in and between classes and how this was reflected in the CMS and other media. It became obvious, however, that this represented a rather one-sided view of communication in the study environment. For this reason, a second case study focused on the students' personal media and other types of one-to-one communication, with a particular focus on how communication with fellow students and communication about study-related matters was integrated into the students' general communication practises and how this reflected their participation in the study environment. Due to the relative age of the data, more recent developments in communication technologies are not included in the analysis. For instance, Facebook was still a relatively minor phenomenon at the time of the fieldwork. For this reason, the current analysis should not be seen as a description of students' communication patterns today; the data is not very useful in this regard. It should, rather, be seen as an analysis and discussion of the way in which different sorts of media, due to their technological characteristics and institutional implementations, reflect organisational structures and social networks in the context of the study.

The first case study was initiated at approximately the same time as a single general content management system replaced a range of alternatives at the University of Copenhagen. Previous systems ranged from simple HTML solutions to various off-the-shelf CMS options, but for a range of reasons, it was considered more appropriate to have one single solution across all faculties and departments. This system was called Absalon, and it included a wide array of functionalities, such as an e-mail system, calendar, and a set of course rooms. The latter was basically a dynamic folder directory allowing file sharing and communication in relation to individual courses but also including more advanced functionalities such as tools for creating surveys, tests, and assignments. In practice, Absalon was administratively organised around teaching activities. "Course-rooms" were automatically established in 
relation to individual courses independent of the teachers and would also be archived when activity in the course room was no longer considered relevant. The first case study focused on on-campus activities in relation to these communication facilities. For this reason, the initial approach was based on participant observation and follow-up interviews, in other words, participating in activities on campus and attending class teaching along with different groups of students in order to share their "information environment" and identify the communication practices and breakdowns involved. The cases were chosen with the purpose of comparing study environments with a high ICT integration to studies with low ICT integration. The Eastern European Section at the Department of Cross-Cultural and Regional Studies turned out to be an interesting site in this regard because it comprised a number of studies with different sizes and different levels of ICT integration.

Among these, Balkan Studies, Russian Studies, and Modern Greek studies made for an interesting comparison due to their relative differences in ICT integration and use. Accordingly, I located the lectures taking place at these studies and followed them in order to observe communication during and between classes. Data consisted of field notes and short ethnographic interviews with the students on site, combined with follow-up focus group interviews focusing on themes that emerged during field observation. This involved five focus group interviews with 15 students lasting 40-60 minutes each. Furthermore, I followed the online communications (if any) taking place in relation to these courses. As mentioned above, the main object of observation was communication practices: conventions, roles, and typical themes defining communication during and between classes as well as the integration of various communication technologies into these practices.

The greatest challenge to this approach turned out to be the localisation of graduate students, who seemed to spend less time on campus and needed to be located in different ways. Accordingly, I included two somewhat larger fields of study involving more graduate students, namely the Department of Musicology and the Department of History, which also displayed considerable differences in ICT integration However, my attempts at establishing contact with graduate students at these fields of study yielded no better results, and I had to rely mainly on written feedback from this group of students. This involved $16 \mathrm{e}$-mail interviews of varying length, ranging from short answers to several exchanges of e-mails. During the analysis, it became increasingly clear that the challenge of locating graduate students was probably due to the very design of the case study and that it actually represented a finding in itself. That is, the focus on on-campus activities and communication facilities presented a highly "administrative" understanding of study activities that mainly served to document the study patterns of undergraduate students. Undergraduate students followed several courses together and thus spent significant time together on campus while graduate students seemed to follow specific courses due to their personal interests and focuses of study and did not seem to spend much time on camp. For this reason, the second case study changed the perspective from activities on campus to students' informal networks, with the result that participant observation was replaced by media diaries. The students 
involved in the study were asked to enter information about their mediated communication in semi-structured communication diaries. The media diary has earlier shown to be a non-credible tool for quantitative measures of media practices (Schultz, Block, \& Custer, 1978) but a good tool for documenting how media becomes part of everyday life (Gauntlett \& Hill, 1999). For this reason, media diaries have been applied as a qualitative tool followed up by focus group interviews in which the students explain events and situations appearing in their media diaries. More specifically, I chose to use semi-structured diaries, for which the general parameters were defined in advance but for which the answer categories were left open. For instance, I asked students to record with whom they were communicating in specific situations and their motives for doing so, but I did not limit the answers to specific categories. The predefined parameters included time, medium, people involved, initiative, motive, and place of communication.

The choice of students for the second case study was based on the findings from the previous case study. It involved a comparison of some of the study patterns appearing in the first case study and focused on networks of students who knew each other already. This included four undergraduate students who were following obligatory courses and had a high degree of identification with the class and three graduate students who had just returned from their internship activities. The study activities of this group of students were based solely on individual focuses and interests, and the informal network between them was grounded in personal history. Finally, a group of graduate students who were writing up their final theses was included because the students shared a small "study environment" on campus, where they were given access to working tables, a wireless network, kitchen facilities, and a library. They thus provided an alternative perspective on ICT in the study environment.

The students were asked to fill in the diary over the course of one week and were then contacted for a follow-up focus group interview in order to obtain more knowledge about the background of the communication described in the communication diaries. The process of distributing and collecting the diaries and setting up focus group interviews was unproblematic, and no informants left the study underway. However, certain aspects of the diary were questioned in ways that invite further methodological reflection. First of all, the structure of the diary as described above was constructed on the basis of a more or less implicit understanding of communication as transmission rather than ritual (Carey, 1989) and as discrete acts of communication rather than processes. Thus, the students' attempts at fitting their actual communication practices into the diary revealed a number of "odd sized" communication phenomena such as unintended, passive, and potential communication that represent interesting challenges and analytical perspectives for future diary studies. Furthermore, my attempt to get the students to specify communication with fellow students caused some resistance, as they seldom refer to fellow students in this way but instead deal with them as friends or acquaintances. This actually represents a finding in itself, which I will return to later on. Before I deal with this issue, however, I will describe the relationship between ICT and organisational structure at the Southern Campus. 


\section{Course management systems and the institutional version of campus life}

Over the course of my study, the relationship between ICT and organisational structure manifested itself in various ways. The formal communication roles and relationships in the university context at large are mapped onto the systems provided by the university administration, most notably the course management system. In McPhee's terms, these systems become an expression of the formal organisational structure and the specific functions and roles it defines. Seen the other way around, the use of personal media tends to express more specific social relationships between students and teachers. As more generic communication tools involved in a wide range of exchanges on campus, they instead reflect the organisation as an informal communication network that is continuously reproduced.

As mentioned in the introduction, the study largely confirms other studies in the field regarding the use of ICT and course management systems. That is, the use of the systems is rather limited and tends to focus on one-way communication from teacher to students. However, while this is sometimes explained as a matter of reservations regarding the technology (Hanson, 2009) or as an issue to be solved as teachers and students become more accustomed to the systems (Lonn \& Teasley, 2009), it might just be a reflection of course communication in general. The one-to-many communication taking place online is an extension of the one-to-many communication taking place offline.

This is most obvious with regard to the communication between teachers and students in the teaching situation. This practice is so obvious to most members of the university organisation that we seldom reflect upon the norms that govern it. They have become part of the organisational commonsense embedded in the physical space on campus, that is, the very physical organisation of the teaching situation. For instance, in almost all of the teaching situations I observed, the position of the teacher is singled out by way of a special physical space that seems to be reserved for "the one who talks". This space is typically placed in front of the blackboard and slightly removed from the rest of the people present in the room. The rest of the people in the room are not prevented from talking, but they most often do so with the permission of the person in the privileged position. Furthermore, whereas the teacher most often directs his statements at the entire class, students' statements are most often directed at the teacher alone, with the rest of the students representing a sort of audience. A few interesting exceptions reveal the importance of this physical arrangement and the communicational conventions it expresses. In some cases, for instance, teacher and students change places in the event of student presentations. In these cases, the students seem to assume this privileged communicational position temporarily. In other cases, teacher and students deliberately change the physical arrangement in the classroom before teaching is initiated in order to sit in a circle, with no position being privileged above others.

Just as the arrangement of tables and chairs reflects a particular set of communication norms, "old ICT" is integrated in accordance with the same communication patterns. For instance, in all teaching situations, the blackboard is the domain of the teacher and is used for one-to-many communication whereas handwritten notes are the domain of the individual 
student and serve as a sort of one-person medium. The communication norms governing the situation as such thus also govern the integration of communication technologies, and the CMS is no exception. Insofar as the Absalon course rooms were actually in use, they mainly served as a communication channel from teacher to students. Of all the course rooms I frequented, only one contained posts from students. In all other cases, the teacher uploaded documents and messages for students to download. The communication did not go the other way around. According to the students' own statements, they mainly communicated with each other by way of personal media such as personal e-mail, text messages, and mobile phones and avoided the course rooms. They did recognise one-to-many media such as e-mail lists and course rooms as possible alternatives, but in practice, these were mainly used by the teacher.

In accordance with Orlikowski's structurational view of technology in organisations (Orlikowski, 1992, 2000), this is most likely due to the particular institutional integration of the system and the structures embedded in the design. At a basic level, naming the technology a "course management system" and naming the individual discussion forums "course rooms" reflects a very particular and very institutional understanding of campus life and communication on campus. This is furthermore reflected in the very design of the technology, including the distinction between teachers, students, and administrators with particular communication privileges and understandings of relevant communication. For instance, from this point of view, learning activities are closely tied to the concept of the course and are meant to take place over three-month periods under particular headlines (courses are archived, and communication is made unavailable after the semester has ended), while other types of learning activities are somewhat ignored. In light of this, it is no surprise that both teachers and students apply and perhaps even exaggerate communication patterns from the classroom and move other types of communication to other media.

\section{Personal media and the students' version of campus life}

Accordingly, personal media seem to be a more obvious choice with regard to students' communication on campus. Personal media, in this perspective, includes several communication technologies such as personal e-mails, mobile phone calls, and text messaging. As Lüders points out (Lüders et al., 2010; Lüders, 2008), the distinction between private and public communication can no longer be based on particular communication technologies; personal communication is, rather, a communication genre defined by its non-professionalised and non-institutional characteristics. According to the students' media diaries and their statements in the first case study, mobile phone calls and text messaging are students' absolute preferred types of communication for various reasons. First of all, as became clear during the first round of focus group interviews following the observations on campus, the choice of a specific type of person-to-person contact media seemed to reflect the specific kinds of relationships on campus. In this way, the choice of communication form became an expression of those relationships existing between students and between students and 
teachers. This perspective was further elaborated in media diaries and follow-up interviews, which led to a more critical discussion of how students actually relate to each other on campus, that is, as friends and acquaintances rather than as colleagues.

In terms of forms of communication, choosing a specific medium for contacting a person also implies a statement with regard to the specific relationship in question. Is it of a personal kind, or is it mainly defined within the framework of a specific organisation? During the first round of interviews, the students defined the mobile phone as a personal medium used for personal contacts, while e-mails were considered a more appropriate contact medium for organisational purposes. For instance, one of the first-year students explained how her participation in a committee at her city council required her to check her e-mail. Whereas she otherwise mainly communicated by way of her mobile phone, her participation in the political work required her to adapt to e-mail communication as well. She considered the use of e-mail as part of organisational work whereas she used her mobile phone for personal communication. Along these lines, the students generally stated that they found it more appropriate to contact their teachers by way of e-mails while they contacted their fellow students by way of mobile phones. This is possibly because the teacher-student relationship is defined within the organisational framework of the university campus and thus calls for a rather formal type of contact. However, a few important exceptions revealed the importance of the specific personal relationships in this regard.

Thus, a few students stated that they might contact specific teachers by way of their mobile phone. Characteristically, this statement was followed by a further explanation of the special relationship they had with this particular teacher. Alternatively, some students found it more appropriate to contact fellow students by way of e-mail. For instance, a student from the Open University mentioned this as the most obvious choice. The same student also pointed out that due to her status as an Open University student, her contact with other students was rather limited. As she did not feel she had any personal relationships with the other students, she preferred to contact them by e-mail if she needed to contact them at all.

The choice of contact medium can be seen as an expression of the specific social relationships existing between teachers and students and between students on campus. The exchange of personal contact numbers seems to be an action of great social significance as it marks a change from a "strictly organisational" kind of relationship to a personal relationship. Use of personal media is thus part of the continuous development and maintenance of a personal social network, which is partly reflected and partly augmented by those traces it leaves on the students' personal media. Before I deal with this in more detail, however, I will describe another important aspect of student communication on campus, namely how students deal with the concept of fellow students.

When handing out the media diaries, I explicitly asked the students to mention when they were communicating with fellow students as this was an important aspect of my inquiry. It turned out that this varied among the groups depending on their study situation. 
The graduate students writing up their final theses were least likely to communicate with fellow students (7 pct. of their entries) whereas the graduate students returning from their internships were most likely to communicate with fellow students (28 pct. of their entries). Interestingly, this did not necessarily mean that more of their communication was related to studies; in fact, only 3 pct. of the entries in this group involved communication about studies. This is very likely due to the particular ways in which studies are organised in the three groups of students. The graduate students writing up their final theses had a common work area, and the undergraduate students had a time schedule that could structure study activities in various ways and ensure continuous meetings with other students. The graduate students returning from their internships had neither, and for this reason, they depended more on personal media for coordinating study activities and social activities with fellow students.

However, in the focus group interviews, these numbers were followed up by a critical discussion of the concept of "fellow student" as such. This did not seem to be a relevant category for the students, and some of them seemed almost uncomfortable with the term as they found it too impersonal for the description of certain relationships and too pretentious for the description of others. If they were communicating with another person from their study, this was because this person was a friend and not because this person was a fellow student. Accordingly, the students seemed to distinguish between other students as either friends or non-friends, whereas the category of fellow student was not socially meaningful. Indeed, this sheds new light on the course management system and its basic design rationale. By defining the course as a primary frame of communication, it assumes that students have a "professional" motivation for communicating with other students, that is, that students in a class view each other as colleagues and communicate on these grounds. According to the students' statements, however, they might instead see the campus as a public space involving several social networks of which they belong to some but not to others. In this way, the course management system might serve as a source of course information, but personal media remains the most relevant way of communicating with other students.

Generally speaking, while the course management systems represent a rather institutional version of campus life and a communication tool controlled primarily by the teacher, students communicate with other students by way of personal media. This seems to be due to the particular way in which students deal with each other: as friends and acquaintances rather than as colleagues. The choice of a specific contact medium reflects social relationships on campus, and personal media can thus be seen as an alternative perspective on the organisational structure on campus, reflecting the social networks between students.

\section{Personal media as an alternative reflection of organisational structure}

As described in the previous section, communication with fellow students seems to be closely related to the process of acquiring friends and establishing a personal network during the study. To judge from the students' statements above, personal media is an important 
aspect of this process since becoming friends with a fellow student also implies integrating him or her into a communication network of friends defined by personal media.

The interaction of the students participating in present case studies thus implies the development of an informal personal network that also tends to be reflected in a student's personal contact details such as his or her phone directory or their MSN account. For instance, the graduate students - in particular those returning from their internships - had more contact details for fellow students than did the undergraduate students. This might partly have to do with the very design of the communication technologies in question. That is, each act of communication may on one hand permit a closer personal relationship while, on the other hand, leaving a "technological trace" in the form of contact details that imply the possibility of later communication. The informal personal network thus grows from concrete acts of communication while the technological memory of the personal media may add historicity (keeping earlier communication for later use) and "resumability" (contact details are stored so that the communication can be easily resumed). These particular design features can be regarded as an alternative representation of organisational structure compared with the course management system's representation of the organisation.

Furthermore, the contact information stored on the students' devices is ultimately a product of their history of interaction and differs fundamentally from the course management system involved in the study. Relevant members of a course room in Absalon are institutionally defined as those students enrolled in a course, thereby encompassing everyone from an institutional point of view, but the composition of the list of course room members says little about actual interaction between students following a particular course. In contrast, the phone directory of an individual student is centred on the individual's interaction history. It thus defines a particular group of people and most likely provides an incomplete picture of the organisational structure. However, pointing back to a history of actual interactions, the phone directory possesses more social relevance. It comprises a set of genuine communication possibilities, whereas the communication possibilities of the Absalon course room often remain hypothetical and rather abstract. They are available in principle, but there is no social motivation for using them.

\section{Conclusion: communication technologies and organisational structure}

In the current study, it has become obvious that different communication technologies within the organisation reflect different aspects of its organisational structure. In accordance with Orlikowski's structurational view of technology in organisations, this can be explained as a consequence of the specific institutional implementation of the technology and can be explained as specific designed features and material properties of the technologies in question. As far as institutional implementation is concerned, the course management system is implemented in accordance with an administrative and institutional perspective of campus life whereas the personal media reflects the students' informal network. In terms of the 
designed features, the course management system's design rationale provides its users with tools that allow them to do the same things online as they would otherwise do in class: distribute course plans and texts, take tests, have discussions, and so on. The personal media represents a much more generic communication tool, used in a wider range of communication contexts. It does not map any communication context in particular but instead adds a technological memory that represents an alternative representation of communication practices.

Of course, it is difficult to say whether it is the institutional implementation or the material properties that has been most decisive in terms of these differences, and it is not within the scope of this article to sort this out or to make more general statements about the relationship between organisational structures and particular communication technologies. However, the analysis points to ways in which students' use of social and personal communication technologies may inform the future design and implementation of course management systems. While many CMS solutions map an ideal teaching situation by creating a virtual "double" of the class, the students' use of social and personal media imply another approach, one in which communication technologies are instead used to visualise the students' interaction history, making it available for later interactions. These features may well serve organisational and educational purposes and could potentially help bridge the gap between the course management system and the everyday reality of campus life.

\section{References}

Aldrich, H.E. (1972). Technology and organizational structure: a reexamination of the findings of the Aston Group. Administrative Science Quarterly, 17, 26-43.

Bijker, W.E., Hughes, T.P., \& Pinch, T.J. (1987). The Social Construction of Technological Systems: New Directions in the Sociology and History of Technology. Cambridge, Mass.: MIT Press.

Blau, P.M., Falbe, C.M., McKinley, W., \& Tracy, P.K. (1976). Technology and organization in manufacturing. Administrative Science Quarterly, 21, 20-40.

Boyd, D.M., \& Ellison, N.B. (2008). Social network sites: definition, history, and scholarship. Journal of Computer-Mediated Communication, 13, 210-230.

Carey, J.W. (1989). Communication as Culture: Essays on Media and Society. Boston: Unwin Hyman.

Cooren, F., Taylor, J.R., \& Van Every, E.J. (2005). Communication as Organizing: Empirical and Theoretical Approaches into the Dynamic of Text and Conversation. Mahwah, NJ: Lawrence Erlbaum.

Conceptual Foundations 46 (Academic Press 1995).

Fairhurst, G.T., \& Putnam, L. (2004). Organizations as discursive constructions. Communication Theory, 14, 5-26.

Gauntlett, D., \& Hill, A. (1999). TV living: television, culture and everyday life. Film, 315.

Grønning, A. (2011). E-tiviteter som eksamensform. Tidsskriftet Laring Og Medier (LOM), 4(7/8).

Hanson, J. (2009). Displaced but not replaced: the impact of e-learning on academic identities in higher education. Teaching in Higher Education, 14, 553-564. 
Lonn, S., \& Teasley, S. D. (2009). Saving time or innovating practice: investigating perceptions and uses of Learning Management Systems. Computers \& Education, 53, 686-694.

Lüders, M., Proitz, L., \& Rasmussen, T. (2010). Emerging personal media genres. New Media \& Society, 12, 947-963.

Lüders, M. (2008). Conceptualising personal media. New Media \& Society, 10(5), 683-702.

McGill, T.J., \& Hobbs, V.J. (2008). How students and instructors using a virtual learning environment perceive the fit between technology and task. Journal of Computer Assisted Learning, 24, 191-202.

McPhee, R.D. (1985). Formal structure and organizational communication. Structure, 13, 149-177.

McPhee, R.D. (2004). Text, agency, and organization in the light of structuration theory. Organization, 11, 355-371.

Orlikowski, W.J. (1992). The duality of technology: rethinking the concept of technology in organizations. Organization Science, 3(3), 398-427.

Orlikowski, W.J. (2000). Using technology and constituting structures: a practice lens for studying technology in organizations. Organization Science, 11, 404-428.

Paulsen, M.F. (2003). Experiences with learning management systems in 113 European institutions. Educational Technology \& Society, 6, 134-148.

Schultz, D.E., Block, M.P., \& Custer, S.J. (1978). A comparative study of radio audience measurement methodology. Journal of Advertising, 7, 14-22.

Sclater, N. (2008). Web 2.0, personal learning environments and the future of learning management systems. Management, 2008, 2008-2009.

Selwyn, N. (2007). The use of computer technology in university teaching and learning: a critical perspective. Journal of Computer Assisted Learning, 23, 83-94.

Taylor, J.R. (2006). Coorientation: a conceptual framework. F. Cooren, J. Taylor, E.J. Van Every (Eds.), Communication as Organizing (pp. 141-156). Mahwah, N.J.: Lawrence Erlbaum Associates.

Taylor, J.R., Cooren, F., Giroux, N., \& Robichaud, D. (1996). The communicational basis of organization: between the conversation and the text. Communication Theory, 6, 1-39.

Taylor, J.R., \& Robichaud, D. (2004). Finding the organization in the communication: discourse as action and sensemaking. Organization, 11, 395-413.

Taylor, J.R., \& Van Every, E.J. (2000). The Emergent Organization: Communication as Its Site and Surface. Mahwah, N.J.: Lawrence Erlbaum Associates.

Anne Mette Thorhauge

Assistant Professor, PhD

Department of Media, Cognition and Communication

University of Copenhagen, Denmark

thorhaug@hum.ku.dk 